\title{
Association of Chinese Visceral Adiposity Index with Nonalcoholic Fatty Liver Disease in Individuals with type 2 Diabetes Mellitus: A Cross Sectional Study
}

\section{Xunan Wu}

Affiliated Hospital of Jiangsu University

\section{Yujing Sha}

Affiliated Hospital of Jiangsu University

\section{Yanyan Li}

Shanghai Pudong Hospital, Fudan University

\section{Ke Chen}

Affiliated Hospital of Jiangsu University

\section{Yue Qiu}

Affiliated Hospital of Jiangsu University

\section{Panpan Zhang}

Affiliated Hospital of Jiangsu University

\section{Weiping Wei}

Affiliated Hospital of Jiangsu University

\section{Ling Yang}

Affiliated Hospital of Jiangsu University

\section{Dong Wang}

Affiliated Hospital of Jiangsu University

\section{Li Zhao}

Affiliated Hospital of Jiangsu University

Guoyue Yuan ( $\nabla$ yuanguoyue@ujs.edu.cn )

Affiliated Hospital of Jiangsu University

\section{Research Article}

Keywords: Chinese visceral adiposity index, Nonalcoholic fatty liver disease, Visceral adiposity, Type 2 diabetes mellitus

Posted Date: January 9th, 2021 
DOl: https://doi.org/10.21203/rs.3.rs-141104/v1

License: (c) (1) This work is licensed under a Creative Commons Attribution 4.0 International License. Read Full License 


\section{Abstract}

\section{Background}

Chinese visceral adiposity index (CVAI), a reliable indicator of visceral adiposity, was associated with type 2 diabetes mellitus (T2DM) in Chinese population. However, little is known regarding association between CVAl and presence of nonalcoholic fatty liver disease (NAFLD) in T2DM patients. Here, we investigated whether CVAl could identify NAFLD among T2DM patients.

\section{Methods}

A total of 504 T2DM patients were recruited in this cross-sectional survey. Anthropometric and biochemical parameters were measured. NAFLD was diagnosed by abdominal ultrasonography. Binary logistic regression was used to assess association between CVAI and occurrence of NAFLD in T2DM patients. The predictive power of CVAI for NAFLD was analyzed by receiver operating characteristic curve (ROC) method.

\section{Results}

CVAl levels were significantly elevated in T2DM patients with NAFLD $(102.68 \pm 32.34$ vs $133.10 \pm 28.44$, $P<0.001)$. Compared with the lowest CVAl quartile, the highest quartile showed worse metabolic features, including waist circumference, body mass index, liver enzymes and lipid parameters $(P<0.01)$. The incidence of NAFLD among T2DM patients increased significantly across CVAl quartiles $(36.5 \%, 57.9 \%$, $78.6 \%, 86.5 \%$, respectively, $P<0.001)$. Binary logistic regression analysis adjusted for multiple factors showed that the odds ratio of having NAFLD was $9.004(95 \% \mathrm{Cl}, 4.441-18.256)$ in the highest CVAI quartile. ROC analyses showed that the area under the curve of CVAI for diagnosing NAFLD was 0.756 (95\% confidence interval: $0.716-0.792)$, which was better than visceral adiposity index, waist-to-hip ratio and waist-to-height ratio $(P<0.05)$. The cut-off value of CVAl for identifying NAFLD in Chinese T2DM population was 111.975 (sensitivity $=0.761$, specificity $=0.627$ ).

\section{Conclusions}

Elevated CVAI levels were independently associated with presence of NAFLD in T2DM patients. CVAI might be a reliable and convenient index for incident NAFLD in T2DM patients.

\section{Background}

Nonalcoholic fatty liver disease (NAFLD), characterized by hepatic fat accumulation in the absence of excessive alcohol consumption, has become the leading cause of chronic liver disease with a global prevalence of $25.2 \%{ }^{[1]}$. It is well-known that NAFLD is intimately intertwined with insulin resistance (IR), which is also the pathogenesis of type 2 diabetes mellitus (T2DM). A latest meta-analysis demonstrated that prevalence of NAFLD among individuals with T2DM patients was up to approximately $55.5 \%$. T2DM patients complicated with NAFLD are prone to develop advanced fibrosis, cirrhosis and 
hepatocellular carcinoma. Meanwhile, T2DM patients coexist with NAFLD are more susceptible to induce T2DM-associated complications, including macro-and micro-vascular complications ${ }^{[3-6]}$. Thus, it is of great necessity to perform early diagnosing for NAFLD in T2DM patients.

Adiposity, especially visceral adiposity, was recognized as an independent risk factor for both T2DM and NAFLD ${ }^{[7,8]}$. Traditional markers of visceral adiposity such as waist circumference (WC) and body mass index (BMI) were not able to distinguish visceral and subcutaneous fat ${ }^{[9]}$. Therefore, a clinical visceral adiposity index (VAI), based on WC, BMI, triglycerides (TG) and high-density lipoprotein cholesterol (HDLC), was proposed to evaluate visceral fat distribution and dysfunction in Caucasians ${ }^{[10]}$. However, Several studies indicated that diagnostic performance of VAI for visceral adiposity and T2DM was not superior to other visceral adiposity indices (BMI, WC) in Chinese population ${ }^{[11,12]}$, which probably attributed to heterogeneity of body fat distribution across ethnic groups. Asian population are more likely to be visceral obesity at a lower BMI values than other ethnicities ${ }^{[13]}$.

Recently, a novel chinese visceral adiposity index (CVAI), calculated by age, BMI, WC, TG, HDL-C, has been introduced to evaluate visceral adipose distribution and dysfunction in Chinese population ${ }^{[14]}$. Previous researches manifested CVAl was associated with incident hypertension, cardiovascular disease and had

a decent predictive value for diabetes in Chinese population ${ }^{[14-17]}$. However, There has been little research on association between CVAI and presence of NAFLD in T2DM patients. This study aimed to explore association of CVAI with occurrence of NAFLD in T2DM patients and to compare diagnostic performance of CVAI with other visceral obesity indices for NAFLD in T2DM patients.

\section{Methods}

\subsection{Study population}

A total of 504 T2DM patients were recruited in this study from the Department of Endocrinology, Affiliated Hospital of Jiangsu University between May 2018 and July 2020. The study was approved by the Ethics Committee of Affiliated Hospital of Jiangsu University. Written informed consent was obtained from each participant. All participants were not treated with hypolipidemic agents (fibrates or statins) or steatogenic medication (eg, corticosteroids, tamoxifen, amiodarone, methotrexate). Patients with type 1 diabetes, gestational diabetes, other specific types of diabetes, polycystic ovary syndrome, history of alcohol abuse ( $\geq 30 \mathrm{~g} /$ day for men, $\geq 20 \mathrm{~g} /$ day for women), liver disease other than NAFLD (viral hepatitis B/C, autoimmune hepatitis, Wilson's disease or other diseases), chronic renal failure, congestive heart failure, acute infection and malignant tumor were excluded from the study.

\subsection{Anthropometric and biochemical measurements}

Patients were divided into two groups: non-NAFLD and NAFLD groups. Each participant was asked for details of smoking status and alcohol consumption. Body weight and height were measured without 
shoes and in light outdoor clothing, BMI was calculated by dividing weight in kilograms by height in meters squared. WC was measured at the midpoint between the lowest rib and the iliac crest to the nearest $0.1 \mathrm{~cm}$. Waist-to-hip ratio (WHR) was calculated as WC divided by hip circumference. Waist-toheight ratio (WHtR) was calculated as WC divided by body height. Seated blood pressure were measured by trained nurses after a 5 -minute rest. Visceral fat area (VFA) and subcutaneous fat area (SFA) were assessed by a visceral fat device区HDS-2000, Omron, China『using bioelectrical impedance analysis (BIA) method.

Fasting plasma glucose (FPG) levels were determined using the glucose oxidase method; Glycosylated hemoglobin (HbA1c) was measured by high-performance liquid chromatography. Fasting insulin (FIns) levels and fasting c-peptide (FC-P) were measured using chemiluminescence; serum total cholesterol (TC), triglycerides, low-density lipoprotein cholesterol (LDL-C), and HDL cholesterol, alanine aminotransferase (ALT), aspartate aminotransferase (AST), were measured using enzymatic methods; and g-glutamyl transpeptidase (GGT) were measured by enzymatic colorimetric method using L-gammaglutamyl-3-carboxy-4-nitroanilide and glycylglycine substrates.

\subsection{Calculation}

The homeostasis model assessment of insulin resistance (HOMA-IR) was calculated from fasting insulin and glucose levels using the following equation:

$\mathrm{HOMA}-\mathrm{IR}=\mathrm{FIns}(\mathrm{ulU} / \mathrm{ml}) * \mathrm{FPG}(\mathrm{mmol} / \mathrm{L}) / 22.5^{[18]}$.

CVAI, VAI were calculated as follows ${ }^{[14]}$ :

Males: CVAl $=-267.93+0.68$ * age $(\mathrm{y})+0.03$ * $\mathrm{BMI}\left(\mathrm{kg} / \mathrm{m}^{2}\right)+4.00$ * WC $(\mathrm{cm})+22.00$ * $\mathrm{Log} 10 \mathrm{TG}(\mathrm{mmol} / \mathrm{L})$ $-16.32 * \mathrm{HDL}-\mathrm{C}(\mathrm{mmol} / \mathrm{L})$

Females: CVAl $=-187.32+1.71$ * age $(\mathrm{y})+4.23 * \mathrm{BMI}\left(\mathrm{kg} / \mathrm{m}^{2}\right)+1.12$ * WC $(\mathrm{cm})+39.76$ * $\log 10 \mathrm{TG}(\mathrm{mmol} / \mathrm{L})$ -11.66 * HDL-C(mmol/L)

Males: $\operatorname{VAl}=\mathrm{WC}(\mathrm{cm}) /\left[39.68+1.88 * \mathrm{BMI}\left(\mathrm{kg} / \mathrm{m}^{2}\right)\right] * \mathrm{TG}(\mathrm{mmol} / \mathrm{L}) / 1.03 * 1.31 / \mathrm{HDL}-\mathrm{C}(\mathrm{mmol} / \mathrm{L})$

Females: $\mathrm{VAl}=\mathrm{WC}(\mathrm{cm}) /\left[36.58+1.89 * \mathrm{BMI}\left(\mathrm{kg} / \mathrm{m}^{2}\right)\right] * \mathrm{TG}(\mathrm{mmol} / \mathrm{L}) / 0.81 * 1.52 / \mathrm{HDL}-\mathrm{C}(\mathrm{mmol} / \mathrm{L})$

\subsection{Diagnosis}

The diagnosis of T2DM was based on the WHO diagnostic criteria 1999. The diagnosis of NAFLD was determined by LOGIQ-9 abdominal ultrasound (Toshiba Medical Systems Corporation, Tokyo, Japan) which was performed by expert ultrasonographers. Diffuse fatty liver can be defined by the presence of at least two out of three abnormal findings on abdominal ultrasonography: diffusely increased liver near 
field ultrasound echo ("bright liver"), liver echo greater than kidney; vascular blurring; and the gradual attenuation of far field ultrasound echo ${ }^{[19]}$.

\subsection{Statistical analysis}

Statistical analyses were carried out using IBM SPSS Statistics Version22 (IBM Corporation, Armonk, NY, USA). Continuous variables which were normally distributed and categorical variables were presented as means \pm standard deviation and percentage (\%). Meanwhile, continuous variables with a skewed distribution were expressed as the median with interquartile range and logarithmically transformed for statistical analysis. The CVAl quartiles were defined as Q1 (very low, CVAl $\leq 101.01$ ), Q2 (low, CVAl 101.02123.40), Q3 (moderate, CVAl 123.41-146.27), Q4 (high, CVAl $\geq 146.28$ ). The Student's T test and MannWhitney $U$ test were used for comparisons of continuous variables between non-NAFLD and NAFLD groups, whereas the one-way analysis of variance (ANOVA) (Bonferroni method for post hoc testing of normally distributed values) and Kruskal-Wallis test were used for multiple comparisons. Moreover, Categorical variables were compared by chi-square test. Pearson or Spearman's correlations were used to evaluate the correlation between CVAI and clinical measurements. Binary logistic regression analyses were used to detect possible independent risk factors for occurrence of NAFLD in T2DM patients and to determine the significant trend for risk of NAFLD across increasing CVAl quartiles using the lowest CVAI quartile as the reference category. The three adjusting models were as follows: Model 1 was adjusted for gender, smoking status and alcohol drinking. Model 2 was additionally adjusted for systolic blood pressure (SBP), diastolic blood pressure (DBP), HOMA-IR and FC-P; Model 3 was further adjusted for TC, LDL-C. For avoiding over adjustment, we did not include age, BMI, WC, TG, HDL-C in the multivariate logistic regression analysis between CVAI and NAFLD because all of these are components of CVAI. Receiver operating characteristic curve (ROC) analyses were used to compare diagnostic performance of CVAI with other anthropometric indices for NAFLD. A two-tailed $P$ value $<0.05$ was considered statistically significant.

\section{Results}

\subsection{Characteristics of the study participants}

Clinical and biochemical characteristics of the participants were shown in Table.1. The study cohort comprised of 504 T2DM patients with a mean age of $55.77 \pm 10.86$ years, $54.4 \%$ were male. Compared with T2DM patients without NAFLD (non-NAFLD group, $n=177$ ), T2DM patients with NAFLD (NAFLD group, $n=327$ ) showed significantly higher BMI, WC, WHR, VFA, SFA, DBP, ALT, AST, GGT, TC, TG, FIns, FC-P, HOMA-IR, VAI, CVAl and lower age, levels of HDL-C (all $P<0.05$ ). There were no significant differences in gender, smoking status, alcohol drinking, WHtR, SBP, LDL-C, FPG and HbA1c.

Characteristics of the study participants according to CVAI quartiles were summarized in Table.2. The quartile ranges of CVAl in participants were Q1 (very low, CVAI $\leq 101.01$ ), Q2 (low, CVAl 101.02-123.40), Q3 
(moderate, CVAl 123.41-146.27), Q4 (high, CVAl $\geq 146.28$ ). The age, BMI, WC, WHR, WHtR, VFA, SFA, SBP and DBP, showed an increasing trend across CVAl quartiles (all $P<0.05$ ), though there were no significant differences in gender, smoking status and alcohol drinking. What's more, FIns, FC-P, HOMA-IR increased by CVAl quartiles $(P<0.001)$, however, no differences in FPG, HbA1c were observed. Subjects with higher CVAl levels were prone to be obese, to have higher liver enzymes (ALT, AST, GGT) and worse lipid parameters (TG, HDL-C) $(P<0.01)$. Furthermore, incidence of NAFLD in T2DM patients was $36.5 \%, 57.9 \%$, $78.6 \%, 86.5 \%$ across CVAl quartiles $(P<0.001)$, showing an increasing trend (Figure 1$)$.

\subsection{Relationships between CVAI and clinical parameters}

CVAI was positively correlated with age, BMI, WC, WHR, WHtR, VFA, SFA, ALT, AST, GGT, FIns, FC-P, HOMAIR, TC and inversely correlated with HDL-C (all $P<0.001$ ). However, no correlation between CVAl and FPG or HbA1c were observed.(Table.3)

\subsection{Association between CVAl and risk of NAFLD in T2DM patients}

We performed a binary logistic regression analysis in which the presence of NAFLD was designated as the dependent variable. Univariate logistic regression analysis showed that risk of NAFLD increased with per-SD increase in CVAI, VAI, WHR, WHtR, TG, TC, FC-P and decreased with that in HDL-C (all $P<0.05$ ). (Table.4). Futhermore, in comparison with the very low CVAl group, the low, moderate, high CVAl groups were independently associated with the occurrence of NAFLD, with ORs of 2.385 (95\% Cl, 1.433-3.969), $6.421(95 \% \mathrm{Cl}, 3.663-11.256)$ and $11.445(95 \% \mathrm{Cl}, 6.081-21.541)(P<0.01)$ after adjusting for gender, smoking status and alcohol drinking. In the model 2 additionally adjusting for SBP, DBP and HOMA-IR, compared with the lowest quartile of CVAI, the OR value for incident NAFLD in the highest quartile of CVAl was $9.181(95 \% \mathrm{Cl} 4.552-18.515, P<0.01)$. The increasing trend remained significant in the fully adjusted multivariable model (Model 3) [ORs:2.037 (95\%Cl, 1.153-3.599), $5.471(95 \% \mathrm{Cl}, 2.898-10.328)$ and 9.004 $(95 \% \mathrm{Cl}, 4.441-18.256)] .(P<0.05)($ Table.5)

\subsection{Diagnostic performance of CVAI for NAFLD.}

ROC analyses were used to evaluate the diagnostic performance of CVAl, VAl, WHR and WHtR for NAFLD. Area under curve (AUC) of CVAl for diagnosing NAFLD was 0.756 (0.716-0.792), which was also better than VAI, WHR and WHtR (all $P<0.05$ ) (Table 6). The cut-off value of CVAl for incident NAFLD was 111.975 (sensitivity $=0.761$, specificity $=0.627$ ).

\section{Conclusion}


Elevated CVAI levels were independently associated with presence of NAFLD in Chinese T2DM population. CVAI was available easily and had better predictive performance than other visceral obesity indices.

\section{Discussion}

In this cross-sectional study of T2DM patients, CVAl was significantly elevated in T2DM patients with NAFLD. The incidence of NAFLD increased across CVAI quartiles, CVAI was significantly associated with presence of NAFLD after adjusting for confounding risk factors related to NAFLD. Moreover, CVAI was superior to VAl, WHR and WHtR in predicting NAFLD among T2DM patients. Our results supported the use of CVAl as a simple clinical index for identifying NAFLD in Chinese T2DM adults.

Adipose tissue, particularly visceral adipose tissue plays a significant role in glucose and lipid metabolism. Excessive visceral fat accumulation rather than general adiposity is closely associated with IR and is an established risk factor for several metabolic diseases, including NAFLD, prediabetes and diabetes $^{[7,8]}$. It is well-known that computed tomography (CT), magnetic resonance imaging (MRI) and dual-energy X-ray absorptiometry (DEXA) are the gold standard to quantify visceral fat area. However, these three methods are expensive, time-consuming and not routinely selected in general clinical use. Therefore, Finding a simple clinical index to identify visceral fat deposition is of great significance. Recently, Xia et al established a novel clinical index, the chinese visceral adiposity index, using a multivariate regression model in Chinese population. CVAl was calculated by age and components of metabolic syndrome (WC, BMI, TG, HDL-C) and proven to be a reliable predictor of visceral adiposity identified by CT. Besides, CVAI was superior to WC, BMI, VAI in diagnosing visceral adiposity ${ }^{[14]}$. In the current study, we demonstrated that CVAI was positively correlated with visceral fat area measured by visceral fat analyzer using BIA method. Thus, CVAI has potential to be a novel surrogate marker to assess visceral adipose deposition in Chinese population.

In this study, we have demonstrated that CVAI was positively correlated with FIns, FC-P and HOMA-IR, suggesting that CVAI was strongly asssociated with IR, which is consistent with the results obtained by $X i a$ et al. IR would aggravate the progression of NAFLD. On the one hand, IR increased hepatic denovo lipogenesis by upregulating expression of sterol regulatory element-binding protein-1c (SREBP-1c). On the other hand, IR in adipose tissue would impair suppression of lipolysis, resulting in migration of excessive fatty acids to liver ${ }^{[20,21]}$. Recently, A prospective study with 4809 subjects revealed that VAI was a reliable predictor of NAFLD in Chinese population ${ }^{[22]}$. However, There has been little research on association between CVAI and presence of NAFLD among T2DM patients. In current study, we have demonstrated that CVAI was positively correlated with liver enzymes and lipid parameters, CVAI was a simple and convenient clinical index of visceral obesity in predicting NAFLD in T2DM patients.

Recently, Several prospective cohort studies revealed that CVAI was a reliable predictor of prediabetes and diabetes in Chinese population. Subjects with higher CVAl levels showed higher plasma glucose levels ${ }^{[16,}$ ${ }^{17]}$. Nevertheless, no differences in FPG, HbA1c were observed across CVAl quartiles in our study, we did 
not find significant correlation between CVAI and FPG or HbA1c. The main reason for the inconsistency with previous researches may be attributed to the fact that individuals from both non-NAFLD group and NAFLD group received regular hypoglycemic therapy.

The possible mechanisms linking visceral obesity and incidence of T2DM and NAFLD were as follows. First, hypertrophic adipocytes in visceral adipose tissue exhibit a resistance to antilipolytic effect of insulin in obese state. As a consequence, excessive FFA are deposited at ectopic sites ( liver, skeletal muscle, pancreas, etc), resulting in IR in both liver and muscle. In contrast, Subcutaneous adipose tissue is characterized by its storage capacity for energy and plays a significant role in maintenance of insulin sensitivity ${ }^{[23]}$. Second, visceral adipose tissue serves as not only a long-term energy storage but also an active endocrine organ. In the setting of increased adiposity, adipocytes would secrete adipokines and cytokines including leptin, TNF-a, resistin, and angiopoietin-like $2^{[24,25]}$, which act as proinflammatory factor to promote obesity-related metabolic disease.

This study is the first to investigate the association of CVAI with presence of NAFLD in T2DM patients. In our study, CVAl was the independent risk factor for incident NAFLD in T2DM patients. Meanwhile, CVAI had a decent predictive value for NAFLD in T2DM patients. However, Limitations of our study also deserve comment. First, This study utilized abdominal ultrasonography to diagnose NAFLD instead of liver biopsy, which is currently the gold standard to determine NAFLD. Compared with liver biopsy,

ultrasonography is less sensitive, particularly when hepatic fat deposition is $<30 \%[26]$. Second, This study was limited by its cross-sectional design, which could not identify causal relationship between CVAl and occurrence of NAFLD in T2DM patients. Therefore, there is a demand to expand the sample size and conduct a prospective study to explore the predictive power of CVAI for presence of NAFLD in T2DM patients.

\section{Abbrevations}

NAFLD: non-alcoholic fatty liver disease; IR: insulin resistance; T2DM: type 2 diabetes mellitus; WC: waist circumference; BMI: body mass index; VAl: visceral adiposity index; CVAl: chinese visceral adiposity index; WHR: waist-to-hip ratio; WHtR: waist-to-height ratio; VFA: visceral adiposity area; SFA: subcutaneous fat area; BIA: bioelectrical impedance analysis; FPG: fasting plasma glucose; HbA1c:

glycosylated hemoglobin; Flns: fasting insulin; FC-P: fasting c-peptide; TC: total cholesterol; TG: triglycerides; LDL-C: low-density lipoprotein cholesterol; HDL-C: high-density lipoprotein cholesterol; ALT: alanine aminotransferase; AST: aspartate aminotransferase; GGT: g-glutamyl transpeptidase; HOMA-IR: homeostasis model assessment of insulin resistance; SBP: systolic blood pressure; DBP: diastolic blood pressure ; ROC: receiver operating characteristic curve; AUC: area under curve.

\section{Declarations}

\section{Acknowledgments:}


The authors appreciate Wei Yin, Diabetes Specialist Nurse, Affiliated Hospital of Jiangsu University, for help with anthropometric measurements.

\section{Authors' contributions}

Xunan Wu and Yujing Sha conceived and designed the study, analysed the data, performed statistical analysis, prepared figures and tables, wrote original paper and approved the final draft.

Yanyan Li, Ke Chen, Yue Qiu, Panpan Zhang and Weiping Wei verified the data, conducted the investigation, approved the final draft.

Ling Yang and Dong Wang: conceived and designed the study, revised the manuscript and tables, approved the final draft.

Li Zhao and Guoyue Yuan conceived and designed the study, analysed the data, performed statistical analysis, revised the manuscript and tables, approved the final draft.

\section{Fundings}

This study was supported by the National Natural Science Foundation of China

(No. 81870548, 81570721, 82000809), the Social Development Project of Jiangsu

Province (No. BE2018692), the Natural Science Foundation of Jiangsu Province

(BK20191222), the High Caliber Medical Personnel Foundation of Jiangsu Province

(LGY2016053), the Six Talent Peaks Project in Jiangsu Province (2015-WSN-006),

the Scientific Research Projects of Jiangsu Health and Family Planning Commission

(Y2018109), the Fifth“169 project” Scientific Research Project of Zhenjiang City,

Jiangsu Province, the Social Development Project of Zhenjiang City, Jiangsu Province (SH2019041).

\section{Availability of data and materials}

The data that support the findings of this study are available from the corresponding authors upon reasonable request.

\section{Ethics approval and consent to participate}


The study observed the Helsinki Declaration for investigation of human and was approved by the Ethics Committee of Affiliated Hospital of Jiangsu University. Written informed consent was obtained from each participant.

\section{Consent for publication}

Not applicable.

\section{Competing interests}

The authors declare that they have no conflicts of interest.

\section{Author details}

${ }^{1}$ Department of Endocrinology, Affiliated Hospital of Jiangsu University, Zhenjiang, Jiangsu, 212300, People's Republic of China

${ }^{2}$ Department of Endocrinology, Shanghai Pudong Hospital, Fudan University, Shanghai, 200120, People's Republic of China

\section{References}

1. Younossi ZM, Koenig AB, Abdelatif D, Fazel Y, Henry L, Wymer M. Global epidemiology of nonalcoholic fatty liver disease-Meta-analytic assessment of prevalence, incidence, and outcomes. Hepatology. 2016;64:73-84.

2. Younossi ZM, Golabi P, de Avila L, Paik JM, Srishord M, Fukui N, et al. The global epidemiology of NAFLD and NASH in patients with type 2 diabetes: A systematic review and meta-analysis. J Hepatol. 2019;71:793-801.

3. Hossain N, Afendy A, Stepanova M, Nader F, Srishord M, Rafiq N, et al, Independent predictors of fibrosis in patients with nonalcoholic fatty liver disease. Clin Gastroenterol Hepatol. 2009;7:1224-9.

4. Piscaglia F, Svegliati-Baroni G, Barchetti A, Pecorelli A, Marinelli S, Tiribelli C, et al. Clinical patterns of hepatocellular carcinoma in nonalcoholic fatty liver disease: A multicenter prospective study. Hepatology. 2016,63:827-38.

5. Wild SH, Walker JJ, Morling JR, McAllister DA, Colhoun HM, Farran B, et al. Cardiovascular Disease, Cancer, and Mortality Among People With Type 2 Diabetes and Alcoholic or Nonalcoholic Fatty Liver Disease Hospital Admission. Diabetes Care. 2018;41:341-7.

6. Jia G, Di F, Wang Q, Shao J, Gao L, Wang L, et al. Non-Alcoholic Fatty Liver Disease Is a Risk Factor for the Development of Diabetic Nephropathy in Patients with Type 2 Diabetes Mellitus. PLoS One. 2015;10:e0142808. 
7. Ko YH, Wong TC, Hsu YY, Kuo KL, Yang SH. The Correlation Between Body Fat, Visceral Fat, and Nonalcoholic Fatty Liver Disease. Metab Syndr Relat Disord. 2017;15:304-11.

8. Neeland IJ, Turer AT, Ayers CR, Powell-Wiley TM, Vega GL, Farzaneh-Far R, et al. Dysfunctional adiposity and the risk of prediabetes and type 2 diabetes in obese adults. JAMA. 2012;308:1150-9.

9. Pou KM, Massaro JM, Hoffmann U, Lieb K, Vasan RS, O'Donnell CJ, et al. Patterns of abdominal fat distribution: the Framingham Heart Study. Diabetes Care. 2009;32:481-5.

10. Amato MC, Giordano C, Galia M, Criscimanna A, Vitabile S, Midiri M, et al. Visceral Adiposity Index: a reliable indicator of visceral fat function associated with cardiometabolic risk. Diabetes Care. 2010;33:920-2.

11. Zhang M, Zheng L, Li P, Zhu Y, Chang H, Wang X, et al. 4-Year Trajectory of Visceral Adiposity Index in the Development of Type 2 Diabetes: A Prospective Cohort Study. Ann Nutr Metab. 2016;69:142-9.

12. Wang B, Zhang M, Liu Y, Sun X, Zhang L, Wang C, et al. Utility of three novel insulin resistance-related lipid indices for predicting type 2 diabetes mellitus among people with normal fasting glucose in rural China. J Diabetes. 2018;10:641-52.

13. Camhi SM, Bray GA, Bouchard C, Greenway FL, Johnson WD, Newton RL, et al. The relationship of waist circumference and BMI to visceral, subcutaneous, and total body fat: sex and race differences. Obesity (Silver Spring). 2011;19:402-08.

14. Xia MF, Chen Y, Lin HD, Ma H, Li XM, Aleteng Q, et al. A indicator of visceral adipose dysfunction to evaluate metabolic health in adult Chinese. Sci Rep. 2016;6:38214.

15. Li B, Lai X, Yan C, Jia X, Li Y. The associations between neutrophil-to-lymphocyte ratio and the Chinese Visceral Adiposity Index, and carotid atherosclerosis and atherosclerotic cardiovascular disease risk. Exp Gerontol. 2020;139:111019.

16. Xia MF, Lin HD, Chen LY, Wu L, Ma H, Li Q, et al. Association of visceral adiposity and its longitudinal increase with the risk of diabetes in Chinese adults: A prospective cohort study. Diabetes Metab Res Rev. 2018;34:e3048.

17. Wu J, Gong L, Li Q, Hu J, Zhang S, Wang Y, et al. A Novel Visceral Adiposity Index for Prediction of Type 2 Diabetes and Pre-diabetes in Chinese adults: A 5-year prospective study. Sci Rep. 2017;7:13784.

18. Matthews DR, Hosker JP, Rudenski AS, Naylor BA, Treacher DF, Turner RC. Homeostasis model assessment: insulin resistance and beta-cell function from fasting plasma glucose and insulin concentrations in man. Diabetologia. 1985;28:412-19.

19. Fan JG, Jia JD, Li YM, Wang BY, Lu LG, Shi JP, et al. Guidelines for the diagnosis and management of nonalcoholic fatty liver disease: update 2010: (published in Chinese on Chinese Journal of Hepatology 2010; 18:163-166). J Dig Dis. 2011;12:38-44.

20. Buzzetti E, Pinzani M, Tsochatzis EA. The multiple-hit pathogenesis of non-alcoholic fatty liver disease (NAFLD). Metabolism. 2016;65:1038-48.

21. Ferrandino G, Kaspari RR, Spadaro O, Reyna-Neyra A, Perry RJ, Cardone R, et al. Pathogenesis of hypothyroidism-induced NAFLD is driven by intra- and extrahepatic mechanisms. Proc Natl Acad Sci 
U S A. 2017;114: E9172-E9180.

22. 22.Xu C, Ma Z, Wang Y, Liu X, Tao L, Zheng D, et al. Visceral adiposity index as a predictor of NAFLD: A prospective study with 4-year follow-up. Liver Int. 2018;38:2294-2300.

23. Agbim U, Carr RM, Pickett-Blakely O, Dagogo-Jack S. Ethnic Disparities in Adiposity: Focus on Nonalcoholic Fatty Liver Disease, Visceral, and Generalized Obesity. Curr Obes Rep. 2019;8:243-54.

24. 24.Fantuzzi G. Adipose tissue, adipokines, and inflammation. J Allergy Clin Immunol. 2005;115:91120.

25. 25.Tabata M, Kadomatsu T, Fukuhara S, Miyata K, Ito Y, Endo M, et al. Angiopoietin-like protein 2 promotes chronic adipose tissue inflammation and obesity-related systemic insulin resistance. Cell Metab. 2009;10:178-88.

26. 26.de Alwis NM, Day CP. Non-alcoholic fatty liver disease: the mist gradually clears. J Hepatol. 2008;48 Suppl 1:S104-S112.

\section{Tables}

Due to technical limitations, tables are only available as a download in the Supplemental Files section.

\section{Figures}




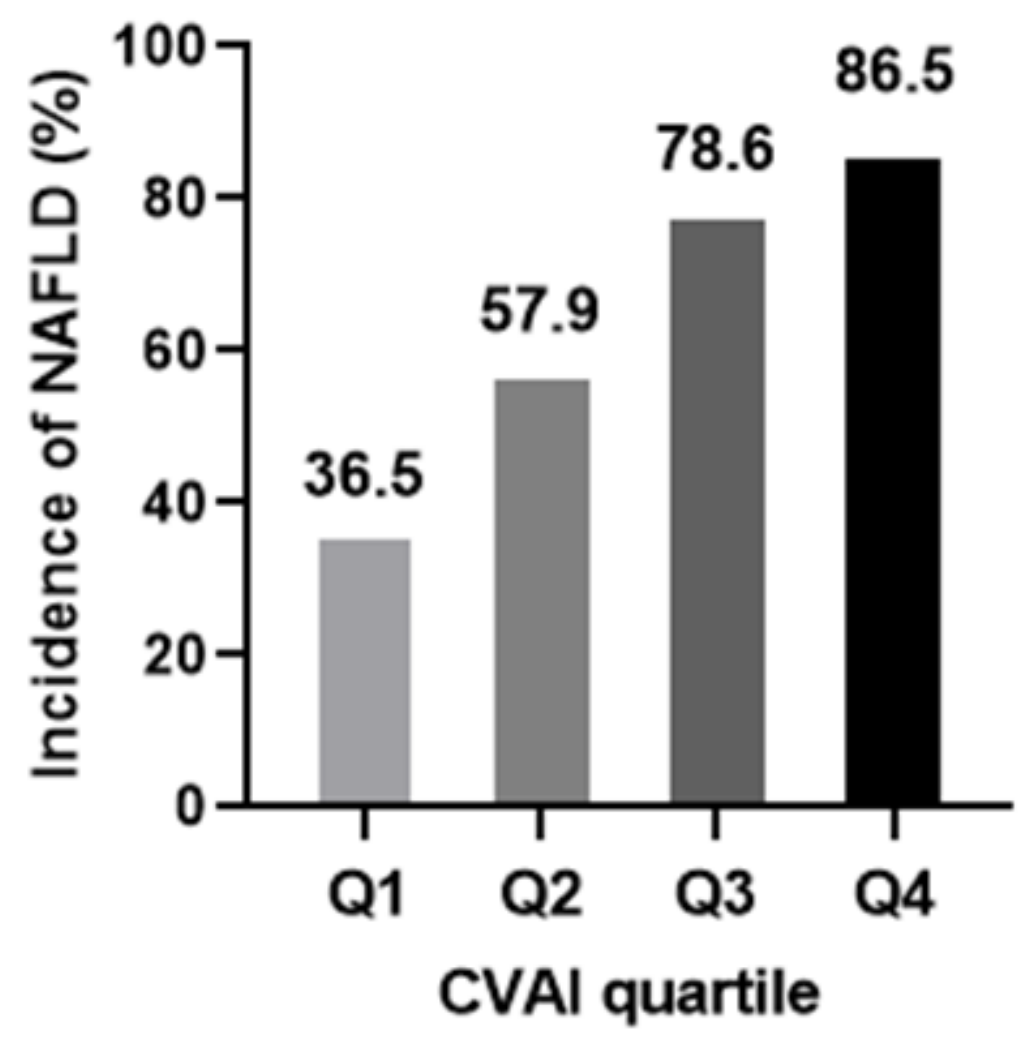

Figure 1

Incidence of NAFLD according to CVAI quartile

\section{Supplementary Files}

This is a list of supplementary files associated with this preprint. Click to download.

- tables.docx 